\title{
A Feedforward Partial Phase Noise Mitigation in the Time-Domain using Cyclic Prefix for CO-OFDM Systems
}

\author{
Youngsun Ha and Wonzoo Chung* \\ Division of Computer and Communications Engineering, Korea University, Seoul 136-713, Korea
}

(Received October 22, 2013 : revised November 14, 2013 : accepted November 14, 2013)

\begin{abstract}
We propose a blind feedforward phase noise mitigation method in the time-domain for a coherent optical orthogonal frequency division multiplexing (CO-OFDM) systems. By exploiting the redundancy of the cyclic prefix $(\mathrm{CP})$, the proposed scheme acquires the overall phase noise difference information during an OFDM block and attempts to mitigate the phase noise in the time domain using a linear approximation. The proposed algorithm mitigates common phase error (CPE) and inter-carrier-interference (ICI) due to phase noise simultaneously, improving the system performance, especially when decision-directed equalizers are used. The simulation results demonstrate the effectiveness of the proposed feedforward phase noise mitigation approach in time domain.
\end{abstract}

Keywords: Coherent optical orthogonal frequency division multiplexing, Phase noise, Optical communication
systems
OCIS codes : $(060.0060)$ Fiber optics and optical communications; (060.4510) Optical communications

\section{INTRODUCTION}

Coherent optical orthogonal frequency division multiplexing (CO-OFDM) is a highly effective technique to achieve highspeed optical communication systems for its high chromatic dispersion (CD) and polarization-mode dispersion (PMD) tolerance $[1,2]$. However, CO-OFDM is vulnerable to the laser phase noise which destroys the orthogonality of the subcarriers. This loss of orthogonality results in inter-carrierinterference (ICI) and common phase error (CPE) in all subcarriers [3]. Since this distortion significantly degrades the system performance, various phase noise compensation techniques have been proposed.

These have mostly focused on frequency-domain phase noise mitigation, i.e., after removing the cyclic prefix (CP) and applying a fast Fourier transform (FFT), where pilot signals or decision direction (DD) methods are available. As mitigation of ICI requires sophisticated methods that incur considerable (indeed, prohibitive) computational expense [4], practical phase compensation studies have mainly considered only CPE mitigation based on either DD methods or high-order-statistics (HOS) blind-adaptive algorithms [5, 6].

In [7] a simple partial ICI mitigation approach has been considered. Using the CPE estimations from the post-discrete Fourier transform (DFT) signals, the algorithm linearly approximates the phase noise in an OFDM block and compensates for it in the frequency domain by convolving of the DFT version of the linear phase model [7]. Although this algorithm is, as far as we know, the simplest algorithm for reducing ICI distortion, it requires accurate CPE estimation from the corrupted data by the phase noise and post convolution processing cost due to its structure. Since the phase noise is a scalar multiplication in the time domain, a direct time-domain correction would be preferable.

In this paper, we propose a non-data-aided feedforward time domain phase noise mitigation scheme that reduces both CPE and ICI by exploiting the redundancy of the cyclic prefix (CP) structure. The $\mathrm{CP}$ is a copy of the last few samples of an OFDM block, which are inserted at the beginning of the block to sustain sub-carrier orthogonality over multipath channels. At the receiver, the $\mathrm{CP}$ structure guarantees that certain received samples match in the absence of phase noise. Based on this property, the phase difference between the beginning and the end of an OFDM block can be roughly estimated. We utilize this phase difference to compensate for ICI and CPE. Especially for systems with a DD equalizer,

\footnotetext{
*Corresponding author: wchung@korea.ac.kr

Color versions of one or more of the figures in this paper are available online.
} 
the proposed feedforward method alleviates the adverse effects of phase noise distortion on the DD equalizer and improves the overall performance.

The remainder of this paper is organized as follows. In Section 2, we describe the discrete time baseband OFDM system and the properties of the CP. In Section 3, we describe the proposed phase noise mitigation algorithm. The numerical results are shown in Section 4, and a conclusion is given in Section 5 .

\section{SYSTEM MODEL}

Let us consider a discrete time baseband CO-OFDM system model, where the $m$-th OFDM symbol consists of $N$ QAM symbols $s_{m}(0), \cdots, s_{m}(N-1)$, and is processed by an inverse DFT (IDFT). At the beginning of the IDFT processed signal block, denoted by $x(0), \cdots, x(N-1)$, the last $P$ samples are inserted and these inserted $P$ samples are called the cyclic prefix (CP). Let $z_{m}(k)$ for $k=-P, \cdots,-1,0, \cdots, N-1$ denote the baseband signal after the insertion of the CP. We then have

$$
z_{m}(k)=z_{m}(k+N)=x_{m}(k+n), k \in-P, \cdots,-1 .
$$

The purpose of $\mathrm{CP}$ is to maintain the orthogonality of the sub-carriers after multi-path channel transmission and the length of the $\mathrm{CP}, P$, should be greater than the channel length. After passing through the optical channel, assuming proper synchronization, the received signal corresponding to the $m$-th OFDM symbol in the presence of laser phase noise is modeled as follows [8]:

$$
r_{m}(k)=z_{m} \otimes h_{m}(k) \cdot e^{j \phi_{m}(n)}+n_{m}(k),
$$

for $\quad k=-P, \cdots-1,0,1 \cdots N-1$, where $Z_{m} \otimes h_{m}$ denotes convolution of the transmitted OFDM symbol $z_{m}(k)$ and the baseband optical channel transfer function $h_{m}(k)$, and $n_{m}(k)$ denotes the zero-mean additive white Gaussian noise (AWGN) with variance $\sigma^{2}$. The optical fiber nonlinearity is not considered here in order to focus on the impact of the phase noise, which is consistent with the analysis described in other previous studies $[3,9]$. The phase noise $\phi_{m}(k)$ for $k=-P, \cdots, N-1$ is modeled as an Wiener process with variance $2 \pi \beta T$, where $\beta$ is the laser linewidth and $T$ is the sample period [10]. In the absence of phase noise and AWGN, we have

$$
r_{m}(-1)=r_{m}(N-1)
$$

as far as the $\mathrm{CP}$ length is set to meet the fundamental requirement of an OFDM system [11]. Note that in the presence of phase noise we can extract the phase difference between $r_{m}(-1)$ and $r_{m}(N-1)$ using this property,

$$
\arg \left\{r_{m}(N-1) / r_{m}(-1)\right\}=\phi_{m}(-1) .
$$

We will exploit this to estimate and compensate for the phase noise in the OFDM blocks.

At the receiver, assuming perfect synchronization under proper distortion monitoring such as in [12, 13], the first $P$ samples, $r_{m}(-P), \cdots, r_{m}(-1)$, i.e., those corresponding to the $\mathrm{CP}$, are removed and processed using a DFT. The output $y_{m}(k)$, which corresponds to the source QAM symbol $s_{m}(k)$, is given as

$$
\begin{aligned}
y_{m}(k)= & I_{m, 0} H_{m}(k) s_{m}(k) \\
& +\sum_{n \neq k}^{N} I_{m, n-k} H_{m}(n) s_{m}(n)+w_{m}(k),
\end{aligned}
$$

where $H_{m}$ and $I_{m, n}$ are the channel frequency response and the distortion due to the phase noise, respectively, and $w_{m}(k)$ is the noise in the frequency domain. The phase noise distortion term, $I_{m, n}$, is given by

$$
I_{m, n}=\frac{1}{N} \sum_{k=0}^{N-1} e^{j\left(\frac{2 \pi k n}{N}+\phi_{m}(k)\right)}
$$

The principal phase distortion $I_{m, 0}$ is the CPE and applies for all sub-carriers, and the remaining terms contribute to the ICI. Note that the relative deviation of $\phi_{m}(k)$ is the main factor that results in ICI, as a constant phase offset $\varphi$ does not affect the ICI (but does affect the CPE) as shown by the following equation:

$$
I_{m, n}=e^{-j \varphi} \frac{1}{N} \sum_{k=0}^{N-1} e^{j\left(\frac{2 \pi k n}{N}+\phi_{m}(k)+\varphi\right)} .
$$

\section{PHASE NOISE MITIGATION SCHEME}

Figure 1 shows a block diagram illustrating the proposed phase mitigation scheme. First, let us define an estimator for the phase noise difference between $\phi_{m}(-1)$ and $\phi_{m}(N-1)$ as

$$
\phi_{m}^{\Delta}:=\arg r_{m}(N-1)-\arg r_{m}(-1) .
$$

Assuming that the first OFDM block $(m=0)$ is a pilot block for channel equalization, we set $\phi_{0}^{\triangle}=0$.

The phase noise sequence $\phi_{m}(0), \cdots, \phi_{m}(N-1)$ is a one dimensional random walk and compensation with a linear line model from $\phi_{m}(0)$ to $\phi_{m}(N-1)$ has been shown to

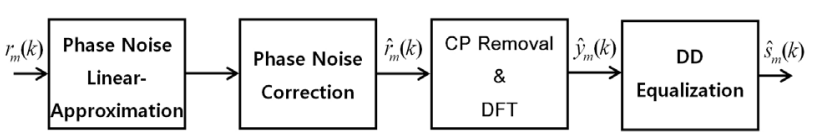

FIG. 1. Detailed block diagram of the proposed algorithm. 
be effective for ICI mitigation [7]. Since we only have the relative phase difference between $\phi_{m}(-1)$ and $\phi_{m}(N-1)$, we approximate the phase noise sequence $\phi_{m}(0), \cdots, \phi_{m}(N-1)$ with the following linear model denoted by $\hat{\phi}_{m}(k)$ with respect to a phase offset $\varphi_{m}$ (if $\phi_{m}(-1)$ is available, $\varphi_{m}$ is set to $\left.\phi_{m}(-1)\right)$, i.e.,

$$
\hat{\phi}_{m}(k)=\varphi_{m}+\frac{k+1}{N} \phi_{m}^{\Delta}, k=0, \cdots N-1
$$

as shown in Fig. 2. This is the optimum mean square error (MSE) interpolation for the phase noise [10]. The phase-compensated signal, denoted by $\hat{r}_{m}(k)$, is given by

$$
\hat{r}_{m}(k)=r_{m}(k) e^{-j \hat{\phi}_{m}(k)}, \quad k=0, \cdots N-1
$$

Regardless of the value of $\varphi_{m}$ (as observed in (9)), this compensation reduces the relative deviation of the phase noise and mitigates the ICI distortion. However, the choice of $\varphi_{m}$ becomes important for CPE. The CPE for the compensated OFDM symbol is given by

$$
\hat{I}_{m, 0}=\frac{1}{N} \sum_{n=0}^{N-1} e^{j\left(\phi_{m}(n)-\frac{n+1}{N} \phi_{m}^{\Delta}-\varphi_{m}\right)}
$$

Assuming mild phase noise, the CPE is can be approximated in the following manner [3]

$$
\begin{array}{r}
\hat{I}_{m, 0} \approx e^{j \frac{1}{N} \sum_{n}\left(\phi_{m}(n)-\frac{n+1}{N} \phi_{m}^{\Delta}-\varphi_{m}\right)} \\
=e^{j\left(\frac{1}{N} \sum_{n} \phi_{m}(n)-\frac{N+1}{2 N} \phi_{m}^{\Delta}-\varphi_{m}\right)}
\end{array}
$$

Note that if $\varphi_{m}=\phi_{m}(-1)$ holds, this approximation reduces the CPE as well (refer to Fig. 2), for we have

$$
\frac{1}{N} \sum_{k=0}^{N-1}\left(\phi_{m}(k)-\phi_{m}(-1)\right) \geq \frac{N+1}{2 N} \phi_{m}^{\Delta}
$$

Given that $\phi_{m}(-1)$ is not available, we use the following approximation

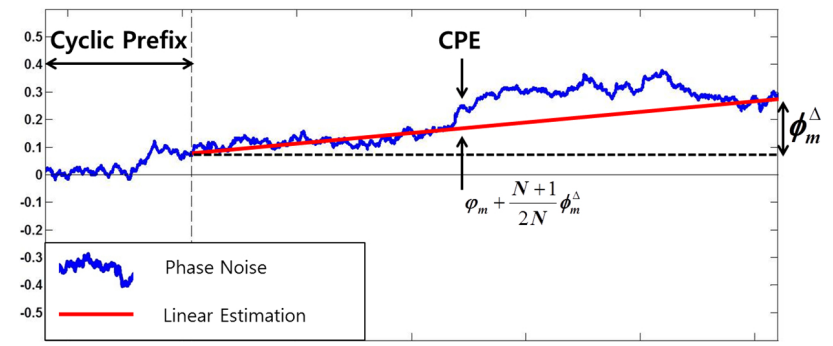

FIG. 2. Phase noise and linearly estimated phase noise.

$$
\varphi_{m}=\sum_{n=0}^{m-1} \phi_{n}^{\Delta}, m=1,2, \cdots N_{f}
$$

where $N_{f}$ denotes the size of the OFDM frame. We then have,

$$
\phi_{m}(-1)-\varphi_{m}=\sum_{n=0}^{m-1}\left(\phi_{n}(-1)-\phi_{n}(-P)\right)
$$

As $m$ increases the accumulated cyclic prefix phase noise becomes significant and the CPE may increase. This problem can be resolved with the help of a DD equalizer. Since the phase offset grows for each $m$, an adaptive DD equalizer can track this increasing phase offset, treating it as a timevarying channel. An estimate of CPE $\hat{I}_{m, 0}$, denoted by $\tilde{I}_{m, 0}$, is given by

$$
\tilde{I}_{m, 0}=\frac{1}{N} \sum_{k=0}^{N-1} \frac{\hat{y}_{m}(k) / H_{m}(k)}{D\left(\hat{y}_{m}(k) / H_{m}(k)\right)}
$$

By updating the DD equalizer to compensate this CPE at every $m$, we can set $\varphi_{m}=0$. Since this approach is simpler and less computationally expensive, it is used for performance evaluation in the following simulation sections. Note that the proposed algorithm does not require past CPE values in the time domain or re-computation of the output symbols as was used in [7].

\section{NUMERICAL RESULTS}

We consider a $21.4 \mathrm{~Gb} / \mathrm{s}$ CO-OFDM system based on 16-QAM signals using 1024 subcarriers $(N=1024)$ with a cyclic prefix of $P=256$ and 32 symbols $\left(N_{f}=32\right)$ per OFDM frame. The pilot symbols were used at the beginning of the frame for channel frequency response estimation. The optical channel response function was assumed to be timeinvariant within one OFDM frame. The transmission length was $500 \mathrm{~km}$ over standard single-mode fiber (SSMF). Fig. 3 shows a comparison of the bit error rate (BER) of the proposed schemes and conventional DD equalizer to compensate for CPE (this is described in (17)) with laser linewidths of $100 \mathrm{kHz}$ and $125 \mathrm{kHz}$. As seen in Fig. 3(a), the proposed scheme provided a $2.7-\mathrm{dB}$ improvement with a BER of $10^{-1.5}$. Fig. 3(b) shows the BER performance of the proposed scheme with a laser linewidth of $125 \mathrm{kHz}$. The conventional DD equalizer encounters the error floor; however, the performance of our algorithm is only slightly degraded in comparison with that of the laser linewidth of $100 \mathrm{kHz}$.

\section{CONCLUSION}

We have described a blind feedforward phase noise mitigation 


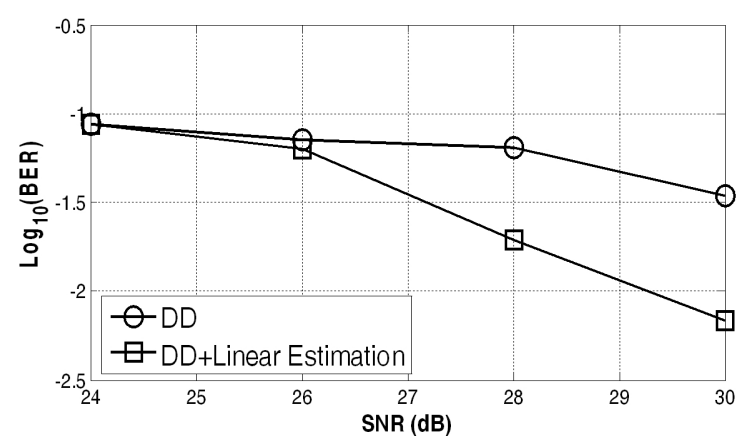

(a)

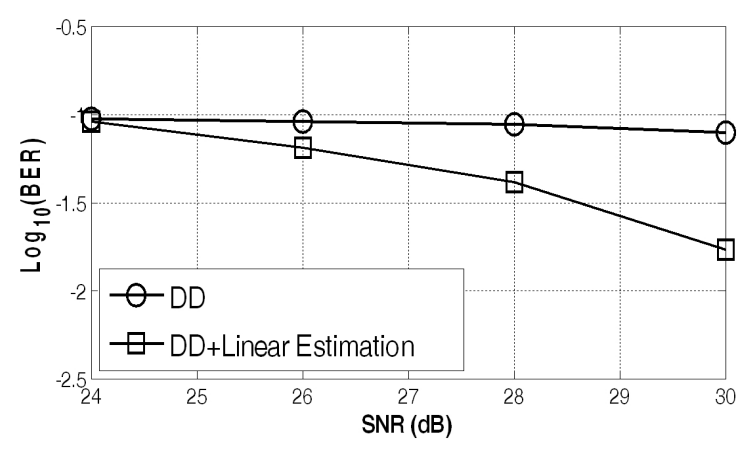

(b)

FIG. 3. The BER performance of the proposed scheme for laser linewidth of (a) 100 and (b) $125 \mathrm{kHz}$.

scheme. The proposed technique reduced both the CPE and ICI by using a linear approximation in the time domain The simulation results demonstrated that the proposed approach effectively improved system performance, especially when DD equalizers were used. In comparision with the conventional phase noise mitigation schemes, the pre-compensation approach of the proposed algorithm significantly improved the system performance with maginal computation load addition.

\section{ACKNOWLEDGMENT}

This work is supported by Basic Science Research Program through the NRF funded by the MEST (2013R1A1A2012626).

\section{REFERENCES}

1. W. Shieh and C. Athaudage, "Coherent optical orthogonal frequency division multiplexing," Electron. Lett. 42, 587-589 (2006).

2. I. B. Djordjevic and B. Vasic, "Orthogonal frequency division multiplexing for high-speed optical transmission," Opt. Express 14, 3767-3775 (2006).

3. W. Shieh, H. Bao, and Y. Tang, "Coherent optical OFDM: theory and design," Opt. Express 16, 841-859 (2008).

4. D. Petrovic, W. Rave, and G. Fettweis, "Effects of phase noise on OFDM systems with and without PLL: Charac-terization and compensation," IEEE Trans. Commun. 55, 1607-1615 (2007).

5. M. E. Mousa-Pasandi and D. V. Plant, "Zero-overhead phase noise compensation via decision-directed phase equalizer for coherent optical OFDM," Opt. Express 18, 20651-20660 (2010).

6. Y. Ha and W. Chung, "Non-data-aided phase noise suppression scheme for CO-OFDM systems," IEEE Photon. Technol. Lett. 25, 1703-1706 (2013).

7. M. E. Mousa-Pasandi and D. V. Plant, "Noniterative interpolation-based partial phase noise ICI mitigation for CO-OFDM transport systems," IEEE Photon. Technol. Lett. 23, 1594-1596 (2011).

8. S. Wu and Y. Bar-Ness, "A phase noise suppression algorithm for OFDM-based WLANs," IEEE Commun. Lett. 6, 535-537 (2002).

9. W. Chung, "A matched filtering approach for phase noise suppression in CO-OFDM systems," IEEE Photon. Technol. Lett. 22, 1802-1804 (2010).

10. P. Rabiei, W. Namgoong, and N. Al-Dhahir, "A non-iterative technique for phase noise ICI mitigation in packet-based OFDM systems," IEEE Trans. Signal Process 58, 5945-5950 (2010).

11. R. K. Martin, J. Balakrishnan, W. A. Sethares, and C. R. Johnson Jr., "A blind adaptive TEQ for multicarrier systems," IEEE Signal Processing Lett. 9, 341-343 (2002).

12. J. Park, W. Chung, J. S. Park, and S. C. Kim, "Non-data aided timing phase recovery scheme for digital equalization of chromatic dispersion and polarization mode dispersion," J. Opt. Soc. Korea 13, 367-372 (2009).

13. K. S. Kim, J. Lee, W. Chung, and S. C. Kim., "An electronic domain chromatic dispersion monitoring scheme insensitive to OSNR using kurtosis," J. Opt. Soc. Korea 12, 249-254 (2008). 